\title{
Ubiquitin-specific peptidase 17 promotes cisplatin resistance via PI3K/AKT activation in non-small cell lung cancer
}

\author{
SHENGCHAO ZHANG, ZHENGLANG XU, JUN YUAN and HAO CHEN \\ Department of Thoracic Surgery, Qingpu Branch Zhongshan Hospital, Fudan University, Shanghai 201700, P.R. China
}

Received November 8, 2018; Accepted November 22, 2019

DOI: 10.3892/ol.2020.11568

\begin{abstract}
The suppression of ubiquitin-specific peptidase 17 (USP17) has previously been found to result in reduced tumorigenesis and invasion of non-small cell lung cancer (NSCLC) cells. However, the functions and underlying mechanisms of USP17 in NSCLC progression remain unclear. In the present study, cisplatin treatment was found to upregulate USP17 expression in a dose-dependent manner. Furthermore, USP17-overexpressing (USP17-OE) NSCLC A549 and H1299 cells were generated for mechanistic studies. The results from the Cell Counting Kit-8 assay revealed increased cell proliferation in USP17-OE cells compared with that of control cells. Moreover, the viability of USP17-OE cells was significantly higher than that of the control cells, when treated with cisplatin. The results of the biochemical studies demonstrated enhanced PI3K and AKT phosphorylation in USP17-OE NSCLC cells, whereas USP17-knockdown decreased these levels of phosphorylation. By contrast, an AKT inhibitor abolished the USP17-mediated enhancement of proliferation. Moreover, suppression of USP17 or the combination of the AKT inhibitor and cisplatin significantly reduced cell viability. Overall, the results of the present study suggest that PI3K/AKT activation is the underlying mechanism of USP17-mediated cisplatin resistance in NSCLC.
\end{abstract}

\section{Introduction}

Lung cancer is the one of the leading causes of cancer-related death worldwide (1), and non-small cell lung cancer (NSCLC) accounts for almost $80 \%$ of these deaths (2). Cisplatin-based chemotherapy is the main treatment used for patients with major NSCLC (2). However, the long-term prognosis for patients with NSCLC still remains poor, with the 5-year survival rate being only $\sim 11 \%$ (3). Patients with NSCLC often

Correspondence to: Dr Hao Chen, Department of Thoracic Surgery, Qingpu Branch Zhongshan Hospital, Fudan University, 1158 East Gongyuan Road, Qingpu, Shanghai 201700, P.R. China E-mail: h.chen@fudan.edu.cn

Key words: ubiquitin-specific peptidase 17, non-small cell lung cancer, AKT inhibitor, cisplatin show an initial positive response to cisplatin treatment, but in many cases this response is not sustained and cancer cells become resistant to the treatment, this has become a major clinical challenge in the treatment of NSCLC (4). Therefore, the identification of novel therapeutic targets in NSCLC treatment that can complement current therapy is urgently required.

The ubiquitin-proteasome system regulates cellular protein levels with specificity and precision to optimize cellular functions $(5,6)$. Moreover, certain studies have demonstrated that ubiquitination can modify protein functions, including the regulation of subcellular localization and protein-protein interactions (5-7). The association between ubiquitin and tumor biology has long been recognized, and the suppression of the proteasome has proven to be effective in the treatment of various types of cancer, such as myeloma and gastrointestinal cancer (6). Deubiquitinating enzymes (DUBs) can reverse protein ubiquitination by digesting ubiquitin chains (8).

Ubiquitin-specific protease 17 (USP17), a DUB, is involved in the regulation of inflammation (9) and cell motility (10), the development of Th17 cells (11) and oncogenesis (12-17). It has been found that USP17 is highly expressed in several types of tumor, including colon, esophageal and cervical tumors (14). In addition, USP17 regulates the Ras pathway by altering the intracellular localization of Ras and other small GTPases, which are crucial regulators of cellular proliferation and migration (10). USP17 is required for the trafficking and oncogenic signaling of mutant epidermal growth factor receptor (EGFR) in NSCLC cells (15). These studies verified the role of USP17 in promoting tumor growth and metastasis. McFarlane et al (16) reported upregulation of USP17 in patients with NSCLC. Moreover, patients with USP17-positive tumors had significantly shorter recurrence-free survival times compared with those with USP17-negative tumors, and USP17 expression was associated with the recurrence of disease at distant sites. In addition, an in vivo study, in which human NSCLC cells were inoculated into nude mice, found that the suppression of USP17 in NSCLC cells inhibited tumor growth and invasion (17). However, the biological function of USP17 that directly regulates NSCLC progression has not been studied fully.

The re-emergence of cancer cells is often due to the activation of survival signals, including increased activation of the PI3K/AKT pathway (18-20), which has been associated with NSCLC progression. The PI3K/AKT pathway is an important pathway downstream of EGFR. Deregulation of this pathway, 
due to gene amplifications, activating oncogene mutations or the loss of PTEN, has been observed in several types of human cancer, such as colorectal, gastric, lung, ovarian and thyroid cancer (20-23).

In the present study, the aim was to explore the functions and underlying molecular mechanisms of USP17 in NSCLC cells. Moreover, the effects of inhibiting of USP17 downstream PI3K/AKT pathway in cisplatin sensitivity of NSCLC cells were also investigated.

\section{Materials and methods}

Cell lines. The human NSCLC A549 and H1299 cell lines were purchased from The Cell Bank of Type Culture Collection of the Chinese Academy of Sciences. A549 cells were maintained in Dulbecco's modified Eagle's medium, and H1299 cells were maintained in RPMI-1640 medium. All media were supplemented with $10 \%$ heat-inactivated fetal bovine serum (FBS; Invitrogen; Thermo Fisher Scientific, Inc.), penicillin $(100 \mathrm{U} / \mathrm{ml})$, and streptomycin $(100 \mu \mathrm{g} / \mathrm{ml})$ in a humidified atmosphere of $5 \% \mathrm{CO}_{2}$ at $37^{\circ} \mathrm{C}$. All cells were confirmed to be free from mycoplasma contamination.

Plasmids and reagents. USP17 short hairpin (sh)RNA and USP17 overexpression lentiviruses were purchased from Hanyin Biotech Co. Polybrene (cat. no. 107689; Sigma-Aldrich; Merck KGaA) was used as an infection reagent. The target sequence for USP17 shRNA-knockdown (KD) was 5'-CTCTTGAGAATGTGCCGAT-3' (the shRNA was packaged into the lentivirus). The negative control (NC) comprised an empty vector without target sequences. To generate stable cell lines, supernatant containing lentivirus ( $\left.1 \times 10^{6} \mathrm{TU}\right)$ was added to A549 and $\mathrm{H} 1299$ cells (1x10 $/$ well), which were subsequently screened with $1 \mu \mathrm{g} / \mathrm{ml}$ puromycin for 2 weeks. Experiments were performed $72 \mathrm{~h}$ after infection.

Reverse transcription-quantitative $(R T-q) P C R$. Total RNA was extracted from cells using TRIzol ${ }^{\circledR}$ (Invitrogen; Thermo Fisher Scientific, Inc.). Total RNA (100 ng) was used for cDNA synthesis using the Stratagene AffinityScript QPCR cDNA Synthesis kit (Agilent Technologies, Inc.). The temperature protocol for the RT step was $5 \mathrm{~min}$ at $65^{\circ} \mathrm{C}, 60 \mathrm{~min}$ at $42^{\circ} \mathrm{C}$ and 15 min at $70^{\circ} \mathrm{C}$. The cDNA samples were diluted 10 -fold with nuclease-free $\mathrm{H}_{2} \mathrm{O}$, of which $2 \mu \mathrm{l}$ was combined with Brilliant III Ultra-Fast SYBR ${ }^{\circledR}$ Green qPCR Master mix (Applied Biosystems; Thermo Fisher Scientific, Inc.). Human RPL13A was used as an internal reference control. The primer sequences were as follows: Human USP17 forward, 5'-GAGATTCTC CGATGTCACAGGC-3' and reverse, 5'-TCCGTCGTGACA ACTCCACCCA-3'; human RPL13A forward, 5'-CTCAAG GTGTTTGACGGCATCC-3' and reverse, 5'-TACTTCCAG CCAACCTCGTGAG-3'. The relative expression of target genes was determined using the $2^{-\Delta \Delta C q}$ method (24). The qPCR cycling conditions comprised an initial denaturation step of $3 \mathrm{~min}$ at $95^{\circ} \mathrm{C}$, followed by 45 cycles at $95^{\circ} \mathrm{C}(10 \mathrm{sec})$ and $58^{\circ} \mathrm{C}$ (45 sec); data were acquired at the end of the annealing/extension phase. Melt curve analysis was performed at the end of each run between $58-95^{\circ} \mathrm{C}$ and the data were analyzed using Microsoft Excel 2013 (Microsoft Corporation).
Cell Counting Kit-8 assay (CCK-8). Cells (5x103/well) were treated with cisplatin $(1 \mu \mathrm{M})$ or MK2206 $(1 \mu \mathrm{M}$; both purchased from Selleck Chemicals) for 0, 24, 48, 72, 96 and $120 \mathrm{~h}$. The CCK-8 assay was conducted according to the kit instructions (cat.no.CK04; Dojindo Molecular Technologies, Inc.). Cells with or without USP17 overexpression (OE) and cells treated with MK2206 were examined. Briefly, cells in logarithmic growth phase were trypsin-digested and resuspended in RPMI-1640 medium. Cells were plated at equal densities $(2,000$ cells $/ 100 \mu \mathrm{l}$ per well) in 96 -well plates and incubated at $37^{\circ} \mathrm{C}$ and $5 \% \mathrm{CO}_{2}$ for continuous detection over a 5-day period. At the beginning of the second day, cell growth was terminated by the addition of $10 \mu \mathrm{l}$ of CCK- 8 solution $(5 \mathrm{mg} / \mathrm{ml})$ to the culture medium. After $2 \mathrm{~h}$, the OD490 values were determined using a microplate reader (BioTek Instruments, Inc.).

Transwell assay. USP17-OE cells and those (2x104/well) treated with MK2206 $(1 \mu \mathrm{M})$ or LY294002 (5 $\mu \mathrm{M}$; both purchased from Selleck Chemicals) for $48 \mathrm{~h}$ were examined. Cells $\left(2 \times 10^{4}\right)$ were detached and resuspended in serum-free medium and seeded in the upper chamber of Matrigel-coated Transwell (precoating with Matrigel at $4^{\circ} \mathrm{C}$ for $60 \mathrm{~min}$ ) inserts with a pore size of $8 \mu \mathrm{m}$. Culture medium containing $10 \%$ FBS as a chemoattractant was added to the lower chamber. After $24 \mathrm{~h}$ of incubation, cells on the upper surface of the insert were gently removed with a cotton swab. Invasive cells (on the lower surface of the insert) were fixed with $4 \%$ paraformaldehyde (Sigma-Aldrich; Merck KGaA) at room temperature for $15 \mathrm{~min}$, stained with crystal violet $(1 \%)$ at room temperature for $60 \mathrm{~min}$, and counted under a microscope (Olympus, CKX31); five random microscopic fields were examined for each insert using magnification, $\mathrm{x} 200$.

Western blot analysis. Total protein was extracted from cells using RIPA buffer (Beyotime Institute of Biotechnology). Protein was quantified using a BCA assay. Protein lysates (50 $\mu \mathrm{g} /$ lane) were separated via $8-10 \%$ SDS-PAGE and transferred onto nitrocellulose membranes. After blocking with $5 \%$ fat-free milk at room temperature for $30 \mathrm{~min}$, the membranes were incubated with primary antibodies (1:500) at $4^{\circ} \mathrm{C}$ overnight. The primary antibodies used were: Goat anti-human USP17 (cat. no. AP5491b; Abgent, Inc.), mouse anti-actin (cat. no. 3700P), anti-human AKT (cat. no. 4685), anti-human phosphorylated (p)-AKT (cat. no. 4060), anti-human p-PI3K (cat. no. 4228) and anti-human PI3K (cat. no. 4257; all purchased from Cell Signaling Technology, Inc.). Following this, membranes were incubated with horseradish peroxidase-conjugated secondary antibodies (1:3,000; cat. nos. 705-035-147, 705-035-150 and 705-035-152; Jackson ImmunoResearch Laboratories, Inc.) at room temperature for $60 \mathrm{~min}$. Immunoreactive proteins were visualized using an enhanced chemiluminescence reagent (EMD Millipore). Relative expression of p-PI3K and p-Akt was normalized to that of actin, and then relative to the normalized total PI3K or Akt values. Quantity One software version 4.6.9 (Bio-Rad Laboratories, Inc.) was used to quantify the relative band intensities.

Statistical analysis. Quantitative variables were compared using one-way ANOVA to compare differences between two 
A

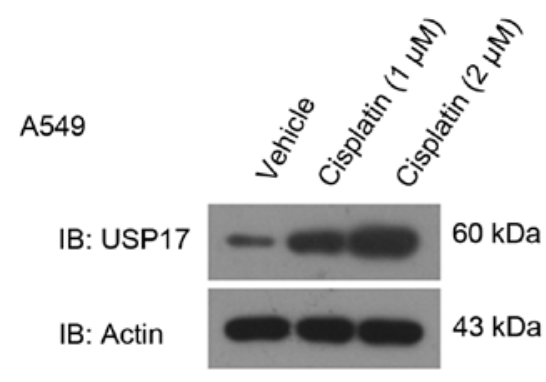

C

$\mathrm{H} 1299$

IB: USP17

IB: Actin
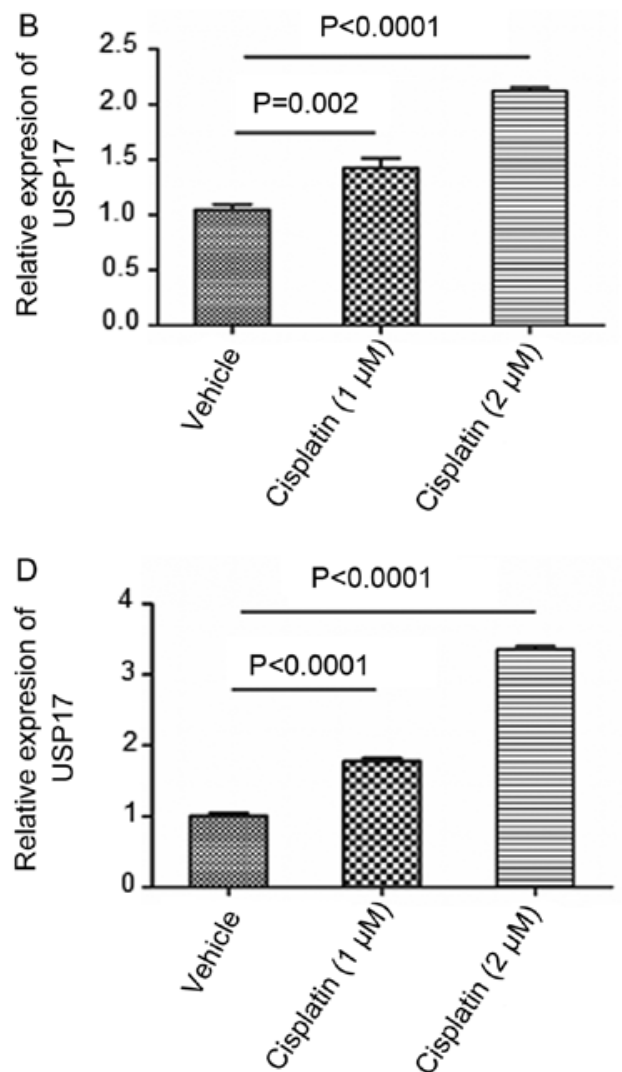

Figure 1. Increased expression of USP17 is observed in non-small cell lung cancer cells treated with cisplatin. Increasing concentrations of cisplatin increased the protein expression level of USP17 in a dose-dependent manner, as shown in (A) western blot images and the (B) corresponding histogram, in A549 cells; and (C) western blot images and the (D) corresponding histogram, in H1299 cells. USP, ubiquitin-specific peptidase.

or more groups, followed by Tukey's test for post-hoc analysis. Statistical analysis was performed using SPSS version 19.0 (IBM Corp.) and GraphPad Prism version 5.0 (GraphPad Software, Inc.). $\mathrm{P}<0.05$ was considered to indicate a statistically significant difference.

\section{Results}

USP17 expression is upregulated in NSCLC cells treated with cisplatin. Western blot analysis revealed increased levels of USP17 in NSCLC cells treated with increasing concentrations of cisplatin, in a dose-dependent manner (vehicle vs. $1 \mu \mathrm{m}$ cisplatin in $\mathrm{A} 549, \mathrm{P}=0.002$, in $\mathrm{H} 1299, \mathrm{P}<0.0001$; vehicle vs. $2 \mu \mathrm{m}$ cisplatin in A549, $\mathrm{P}<0.0001$, in $\mathrm{H} 1299, \mathrm{P}<0.0001$; Fig. 1).

In order to address the functions of USP17 in the response of NSCLC cells to cisplatin treatment, USP17-OE A549 and H1299 NSCLC cells were generated. Both RT-qPCR and western blot analysis demonstrated high expression levels of USP17 in these cell lines, compared with those in the corresponding NC cells (NC vs. OE, $\mathrm{P}<0.001$; Fig. 2).

Overexpression of USP17 increases proliferation and sustains viability in NSCLC cells. In order to investigate the biological functions of USP17 in the transformation of NSCLC cells, a CCK-8 assay was conducted to determine the proliferative ability of A549 (Fig. 3A) and H1299 (Fig. 3B) cells (NC vs. $\mathrm{OE}, \mathrm{P}<0.001)$. Moreover, the viability of USP17-OE cells was significantly higher than that of the control cells when treated with cisplatin ( $\mathrm{NC}$ vs. $\mathrm{OE}, \mathrm{P}<0.001$; Fig. $3 \mathrm{C}$ and $\mathrm{D}$ ). These results demonstrate that the overexpression of USP17 increases proliferation and viability in NSCLC cells, independent of cisplatin treatment.

Overexpression of USP17 enhances PI3K/AKT pathway activation in NSCLC cells. The importance of the PI3K/AKT pathway as a potent survival mechanism in carcinogenesis and tumor metastasis is well established (18-20). In the present study, the effect of USP17 on the activation of the PI3K-AKT signaling pathway in NSCLC cells was investigated. Western blot analysis demonstrated increased p-AKT and p-PI3K levels in USP17-OE NSCLC cells, compared with those of the control cells ( $\mathrm{NC}$ vs. OE, $\mathrm{P}<0.0001$; Fig. 4). This finding suggests the activation of the PI3K/AKT pathway by USP17 as the underlying mechanism of the enhanced proliferation and viability in NSCLC cells.

Inhibition of AKT activation abolishes USP17-mediated enhancement of proliferation and viability in USP17-OE cells. MK2206, an allosteric AKT inhibitor, has been demonstrated to decrease cell proliferation in other types of cancer (25-27). Following treatment with $1 \mu \mathrm{M}$ MK2206, the viability and proliferation of A549-USP17-OE and H1299-USP17-OE cells were significantly decreased (DMSO vs. MK2206, $\mathrm{P}<0.001$; Fig. 5A and B), which further supports the functional association between USP17 and AKT activation. Moreover, the combination of the AKT inhibitor with cisplatin significantly reduced cell viability and proliferation (DMSO vs. MK2206, $\mathrm{P}<0.001$; Fig. 5C and D). 
A

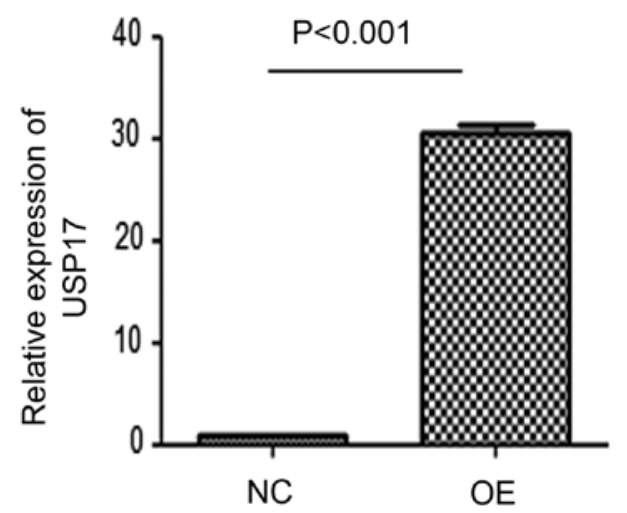

C $\mathrm{A} 549$

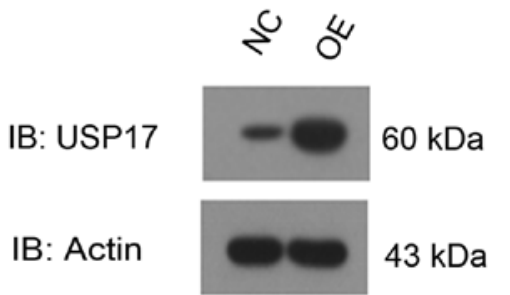

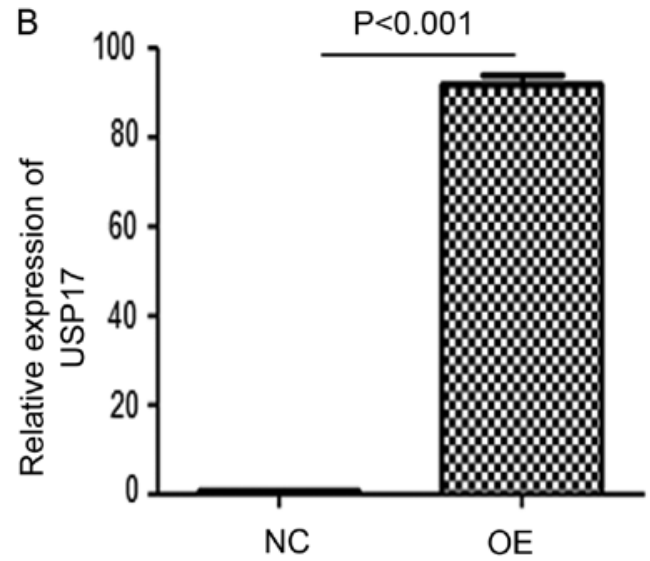

D $\mathrm{H} 1299$

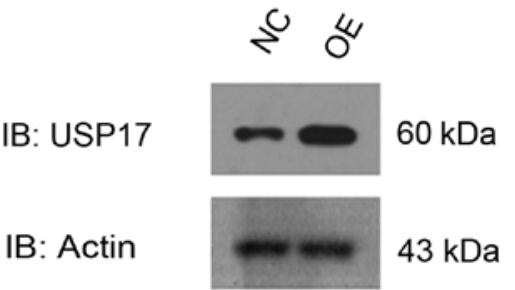

Figure 2. USP17-OE in non-small cell lung cancer cells. Reverse transcription-quantitative PCR analysis of USP17-OE efficiency in (A) A549 and (B) H1299 cells. Western blot analysis of USP17-OE efficiency in (C) A549 and (D) H1299 cells. USP, ubiquitin-specific peptidase; NC, negative control; OE, overexpression.
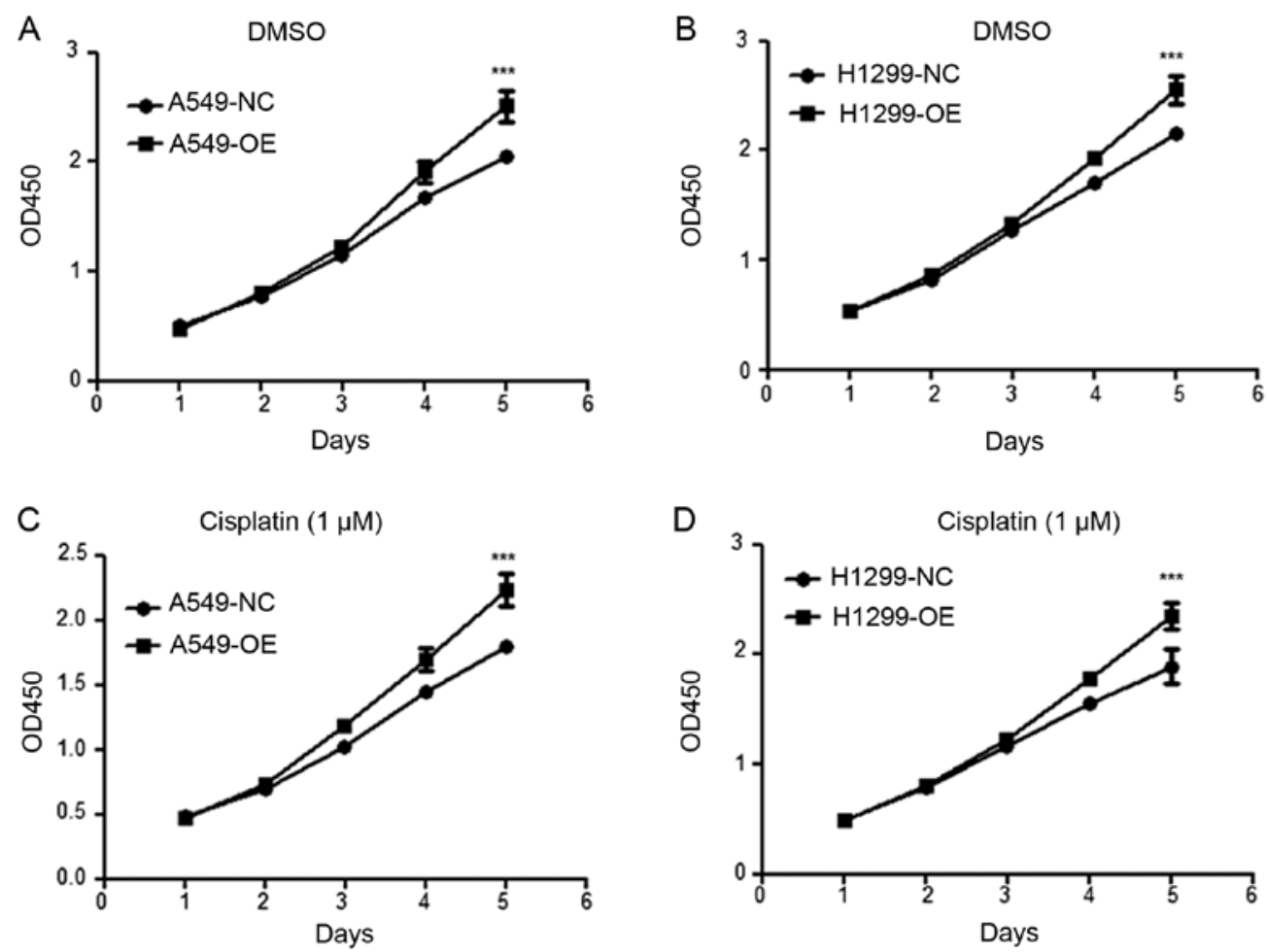

Figure 3. Overexpression of ubiquitin-specific peptidase 17 increases the proliferation and viability of non-small cell lung cancer cells. Cell Counting Kit- 8 analysis comparing USP17-OE with the NC in (A) A549 and (B) H1299 cells treated with DMSO; and (C) A549 and (D) H1299 cells treated with cisplatin $(1 \mu \mathrm{M}) .{ }^{* * *} \mathrm{P}<0.001$ vs. NC. OE, overexpression; NC, negative control; USP, ubiquitin-specific peptidase.

USP17 promotes proliferation and invasion through $P I 3 K / A K T$ activation in NSCLC. Consistent with the findings from the USP17-OE cells, knock-down (KD) of USP17 decreased the protein expression levels of p-AKT and p-PI3K in NSCLC cells (NC vs. KD, P<0.0001; Fig. 6A-F). In addition, the viability of USP17-KD cells was decreased following 

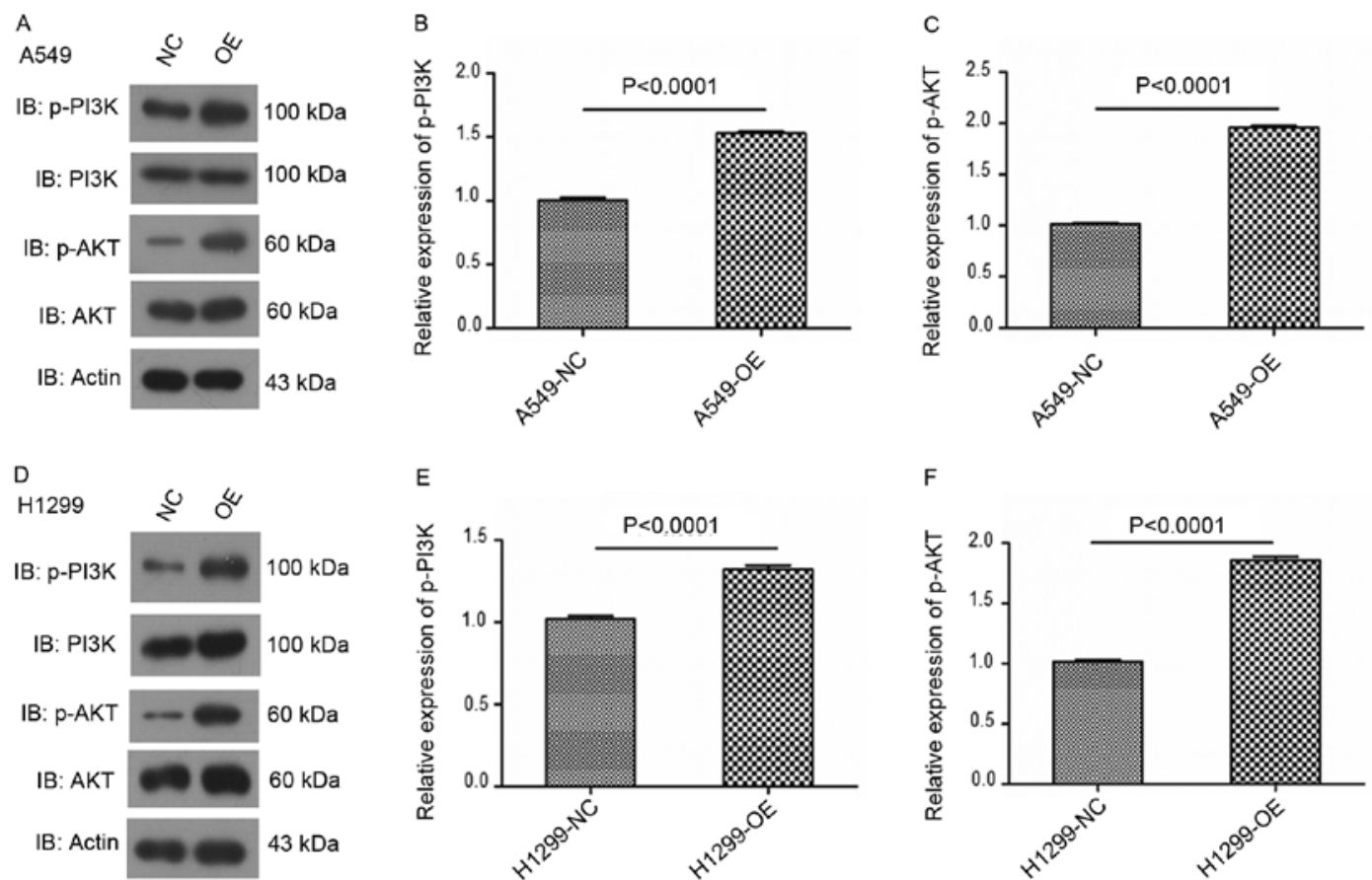

Figure 4. USP17-OE enhances activation of the PI3K/AKT pathway in non-small cell lung cancer cells. Western blot analysis of p-PI3K, PI3K, p-AKT and AKT levels in (A-C) A549 and (D-F) H1299 cells with or without USP17-OE. USP, ubiquitin-specific peptidase; OE, overexpression; NC, negative control; p, phosphorylated.

A

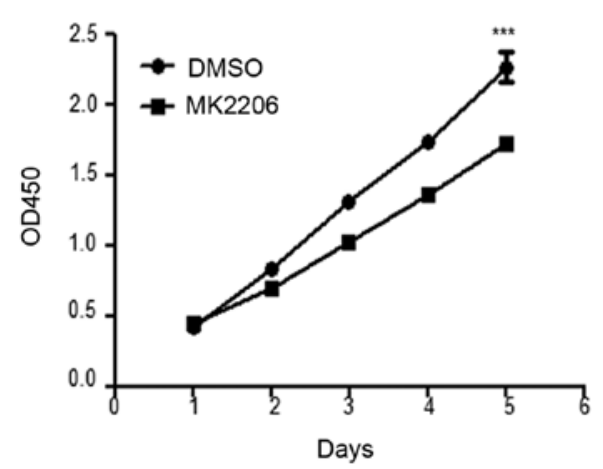

C

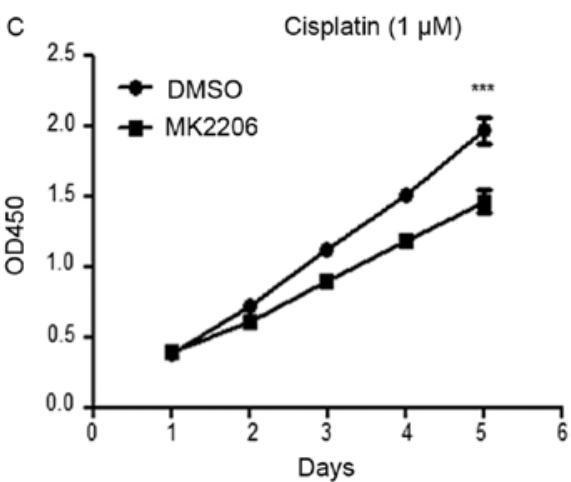

Vehicle
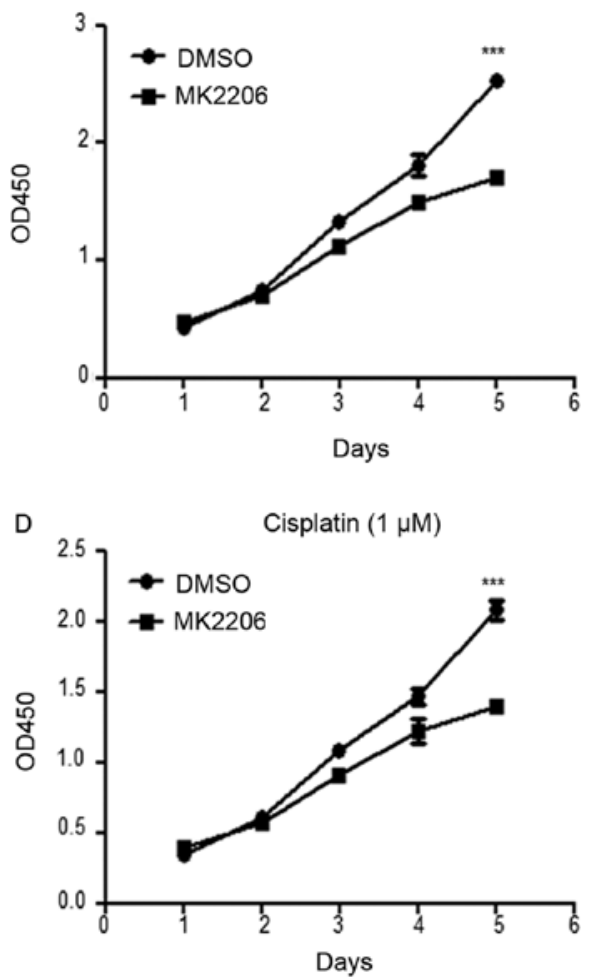

Figure 5. Inhibition of AKT activation abolishes USP17-mediated enhancement of proliferation and viability in USP17-OE cells. Cell Counting Kit-8 assay in USP17-OE A549 (A and C) and H1299 cells (B and D) treated with DMSO control or MK2206, alone or in combination with cisplatin. ${ }^{* * *} \mathrm{P}<0.001$ vs. MK2206. DMSO, DMSO negative treatment control; USP, ubiquitin-specific peptidase; OE, overexpression.

cisplatin treatment ( $\mathrm{NC}$ vs. $\mathrm{KD}, \mathrm{P}<0.001$; Fig. 6G-H). Moreover, the invasive capacity of A549-USP17-OE and H1299-USP17-OE cells was significantly decreased in the presence of MK2206 and the PI3K inhibitor LY294002 (DMSO vs. MK2206, P<0.001; DMSO vs. LY294002, $\mathrm{P}<0.001$; Fig. 7). 

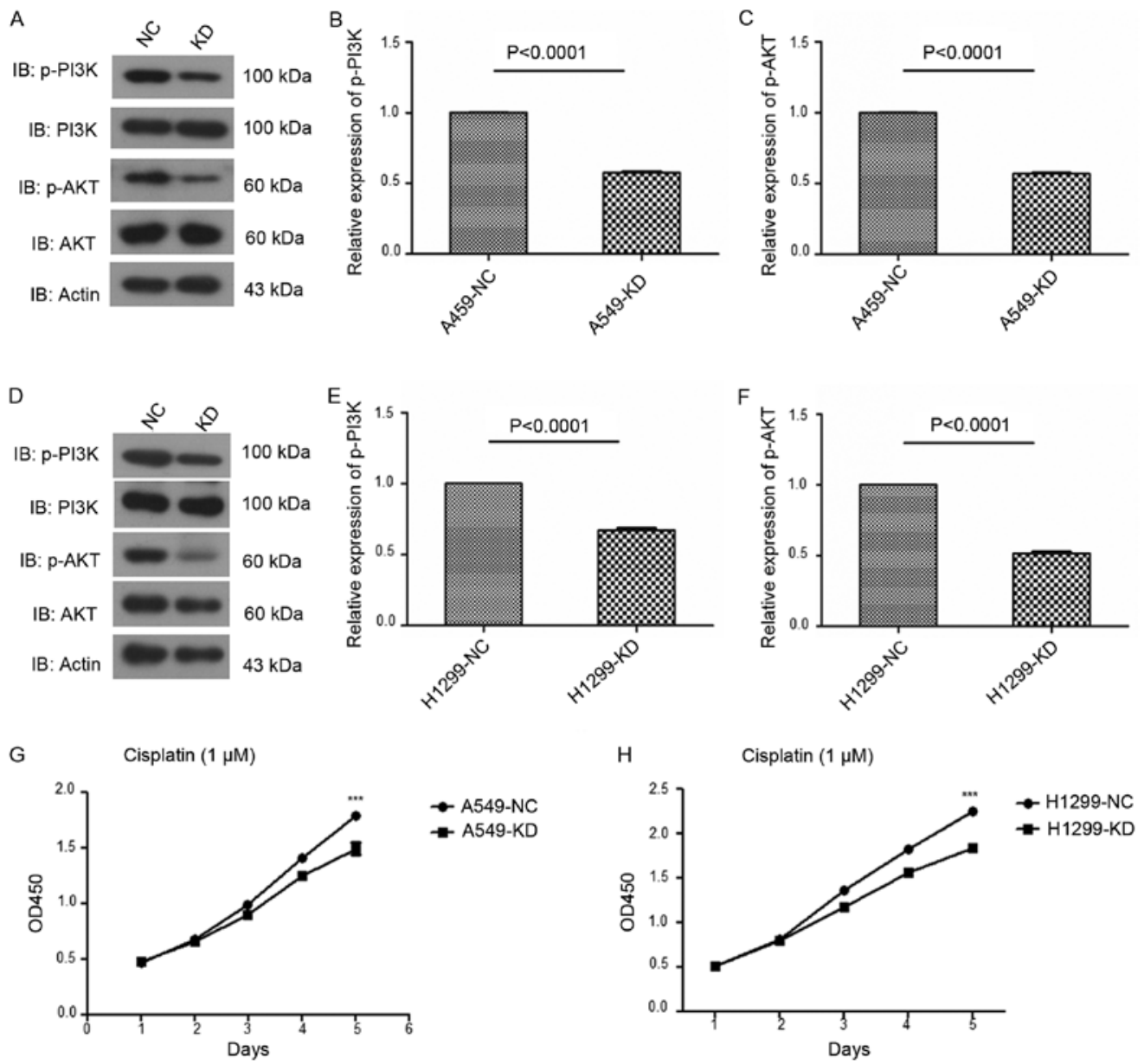

Figure 6. USP17 suppression decreases PI3K/AKT pathway activation and the viability of non-small cell lung cancer cells. Western blot analysis of p-PI3K, PI3K, p-AKT and AKT protein levels in (A-C) A549 and (D-F) H1299 cells with or without USP17 KD. (G) Cell Counting Kit-8 assay in USP17 KD and NC (G) A549 and (H) H1299 cells treated with cisplatin. ${ }^{* * *} \mathrm{P}<0.001$ vs. KD. USP, ubiquitin-specific peptidase; KD, knockdown; NC, negative control; immunoblot; p-, phosphorylated.

A

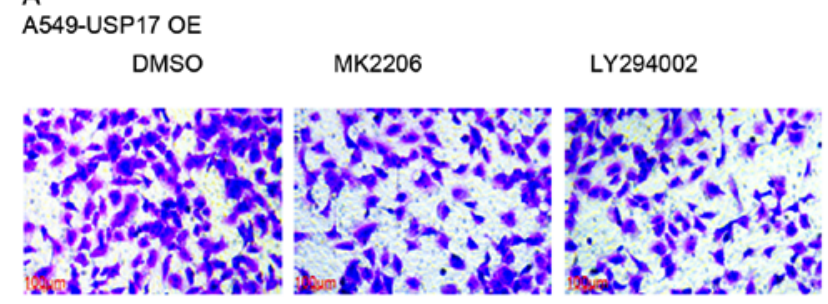

C

H1299-USP17 OE
DMSO

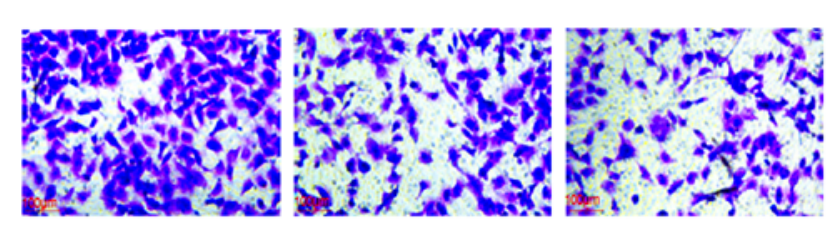

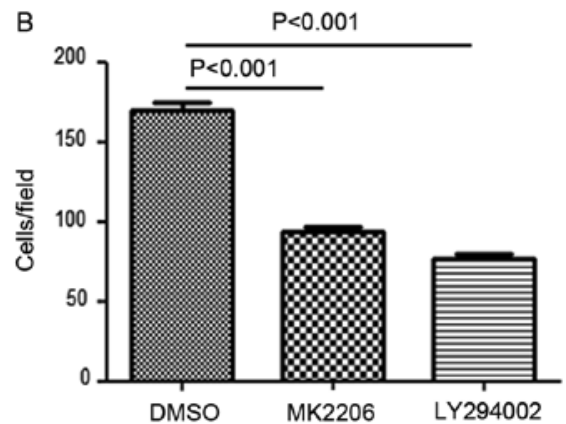

D

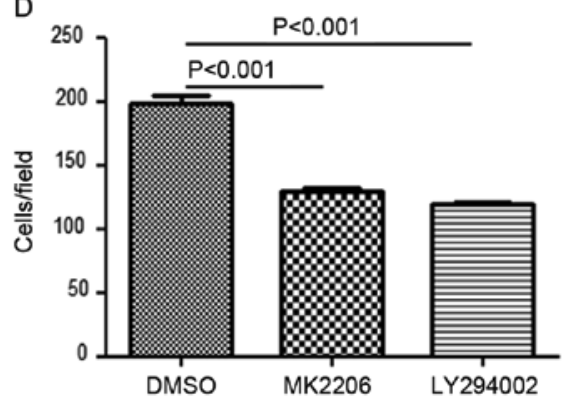

Figure. 7. USP17-induced non-small cell lung cancer cell invasion is mediated by the PI3K/AKT pathway. Transwell assay using USP17-OE (A and B) A549 and (C and D) H1299 cells treated with the DMSO control, MK2206 or LY294002. Magnification, x200; scale bar, $100 \mu$ m. USP, ubiquitin-specific peptidase; $\mathrm{OE}$, overexpression; DMSO, DMSO negative treatment control. 
Overall, the findings of the present study demonstrate the promotion of NSCLC cell proliferation and viability by USP17, via the activation of the PI3K/AKT pathway.

\section{Discussion}

The USP family is one of the five subfamilies of DUB enzymes, which cleave polyubiquitin chains from proteins, a number of studies have reported that USP17 has oncogenic characteristics. Firstly, it has been shown that high levels of USP17 in lung, colon, esophageal and cervical tumor samples promoted G1/S transition and cellular proliferation (14-17). Additionally, it was demonstrated that USP17 was expressed highly in NSCLC tissues, and patients with high levels of USP17 exhibited lower survival rates $(16,17)$. Moreover, USP17 can be induced by cytokines such as interleukin (IL)-4 and IL-6 (28). In the present study, it was found that cisplatin treatment upregulated USP17 expression in a dose-dependent manner. Moreover, increased cell proliferation was found in USP17-OE cells compared with that of control cells, which is consistent with previous studies (14-17). Furthermore, it was demonstrated that the viability of USP17-OE cells was significantly higher than that of the control cells, when treated with cisplatin. In the present study USP17 regulated cisplatin sensitivity in NSCLC. USP17 was initially identified as a regulator of cell viability via signaling pathways associated with cell death in cervical cancer (29).

It was previously reported that USP17 expression could regulate Ras cellular localization and activation, via the deubiquitination of Ras-converting enzyme 1, thereby inhibiting phosphorylation of the downstream kinases, dual specificity mitogen-activated protein kinase kinase and ERK (30). In osteosarcoma, USP17 facilitated cell migration and invasion by deubiquitinating and stabilizing SMAD4 (31). USP17- and Skp1-cullin-1-F-box protein $\beta$ F-box/WD repeat-containing protein 1A-regulated degradation of differentially expressed in chondrocytes protein 1 has been shown to control the DNA-damage response $(32,33)$, thus indicating that the expression of USP17 may be associated with sensitivity to chemotherapeutics, including cisplatin.

In the present study, USP17 was found to promote the growth of NSCLC cells via the activation of the PI3K/AKT pathway. Aberrant activation of the PI3K/AKT pathway is often detected in numerous types of human cancer; hence, targeting this pathway may have therapeutic potential for the management of these tumors (34-37). Emerging evidence indicates that the activation of PI3K/AKT signaling by hypoxia may be a contributing factor to drug resistance in certain types of human cancer, including NSCLC and prostate cancer (38-40). PI3K activation is regulated by various molecules in NSCLC, such as DIX domain-containing protein 1, GRB2-associated-binding protein 2 and microRNAs (41-43).

There are limitations of the present study. Firstly, the functions of USP17 in the response of NSCLC cells to cisplatin treatment require further investigation, specifically using in vivo animal models $(44,45)$. Moreover, as the underlying mechanisms of USP17 in the cisplatin response have not been investigated in this study, it should be examined in the future.

Considerable effort has been made to identify agents that target the activity of DUBs. However, no effective drugs have yet entered into clinical trials. The findings from the present study demonstrate the therapeutic potential of targeting PI3K/AKT pathways downstream of USP17 to prevent NSCLC progression.

\section{Acknowledgements}

Not applicable.

\section{Funding}

The present study was supported by the Shanghai Qingpu District Health and Planning Commission Research Fund (grant no. W2017-21) and the Qingpu District Science and Technology Commission (grant no. QKY2017-03).

\section{Availability of data and materials}

The datasets used and/or analyzed during the current study are available from the corresponding author on reasonable request.

\section{Authors' contributions}

SZ and HC contributed to designing research studies, conducting experiments, acquiring data, analyzing data, providing reagents and writing the manuscript. ZX and JY contributed to conducting experiments and acquiring data.All authors read and approved the final manuscript.

\section{Ethics approval and consent to participate}

Not applicable.

\section{Patient consent for publication}

Not applicable.

\section{Competing interests}

The authors declare that they have no competing interests.

\section{References}

1. Mao Y, Yang D and Krasna MJ: Epidemiology of lung cancer. Surg Oncol Clin N Am 25: 439-445, 2016.

2. Azar FE, Azami-Aghdash S, Pournaghi-Azar F, Mazdaki A, Rezapour A, Ebrahimi P and Yousefzadeh N: Cost-effectiveness of lung cancer screening and treatment methods: A systematic review of systematic reviews. BMC Health Serv Res 17: 413, 2017.

3. Jacobsen MM, Silverstein SC, Quinn M, Waterston LB, Thomas CA, Benneyan JC and Han PKJ: Timeliness of access to lung cancer diagnosis and treatment: A scoping literature review. Lung Cancer 112: 156-164, 2017.

4. Giaccone G: Clinical perspectives on platinum resistance. Drugs 59: 9-37, 2000.

5. D'Andrea A and Pellman D: Deubiquitinating enzymes: A new class of biological regulators. Crit Rev Biochem Mol Biol 33: 337-352, 1998.

6. Weathington NM and Mallampalli RK: Emerging therapies targeting the ubiquitin proteasome system in cancer. J Clin Invest 124: 6-12, 2014.

7. Welchman RL, Gordon C and Mayer RJ: Ubiquitin and ubiquitin-like proteins as multifunctional signals. Nat Rev Mol Cell Biol 6: 599-609, 2005.

8. Nijman SM, Luna-Vargas MP, Velds A, Brummelkamp TR, Dirac AM, Sixma TK and Bernards R: A genomic and functional inventory of deubiquitinating enzymes. Cell 123: 773-786, 2005. 
9. Song H, Tao L, Chen C, Pan L, Hao J, Ni Y, Li D, Li B and Shi G: USP17-mediated deubiquitination and stabilization of HDAC2 in cigarette smoke extract-induced inflammation. Int J Clin Exp Pathol 8: 10707-10715, 2015.

10. de la Vega M, Kelvin AA, Dunican DJ, McFarlane C, Burrows JF, Jaworski J, Stevenson NJ, Dib K, Rappoport JZ, Scott CJ, et al: The deubiquitinating enzyme USP17 is essential for GTPase subcellular localization and cell motility. Nat Commun 2: 259, 2011.

11. Han L, Yang J, Wang X, Wu Q, Yin S, Li Z, Zhang J, Xing Y, Chen Z, Tsun A, et al: The E3 deubiquitinase USP17 is a positive regulator of retinoic acid-related orphan nuclear receptor $\gamma \mathrm{t}$ (ROR $\gamma$ t) in Th17 cells. J Biol Chem 289: 25546-25555, 2014.

12. Borbely G, Haldosen LA, Dahlman-Wright K and Zhao C: Induction of USP17 by combining BET and HDAC inhibitors in breast cancer cells. Oncotarget 6: 33623-33635, 2015.

13. Hu M, Chen H, Han C, Lan J, Xu Y, Li C, Xue Y and Lou M: Expression and functional implications of USP17 in glioma. Neurosci Lett 616: 125-131, 2016.

14. McFarlane C, Kelvin AA, de la Vega M, Govender U, Scott CJ, Burrows JF and Johnston JA: The deubiquitinating enzyme USP17 is highly expressed in tumor biopsies, is cell cycle regulated, and is required for G1-S progression. Cancer Res 70: 3329-3339, 2010

15. McCann AP, Smyth P, Cogo F, McDaid WJ, Jiang L, Lin J Evergren E, Burden RE, Van Schaeybroeck S, Scott CJ and Burrows JF: USP17 is required for trafficking and oncogenic signaling of mutant EGFR in NSCLC cells. Cell Commun Signal 16: 77, 2018.

16. McFarlane C, McFarlane S, Paul I, Arthur K, Scheaff M, Kerr K, Stevenson M, Fennell DA and Johnston JA: The deubiquitinating enzyme USP17 is associated with non-small cell lung cancer (NSCLC) recurrence and metastasis. Oncotarget 4: 1836-1843, 2013.

17. Zhang S, Yuan J and Zheng R: Suppression of Ubiquitin-Specific Peptidase 17 (USP17) inhibits tumorigenesis and invasion in non-small cell lung cancer cells. Oncol Res 24: 263-269, 2016.

18. Graff JR, Konicek BW, McNulty AM, Wang Z, Houck K, Allen S, Paul JD, Hbaiu A, Goode RG, Sandusky GE, et al: Increased AKT activity contributes to prostate cancer progression by dramatically accelerating prostate tumor growth and diminishing p27Kip1 expression. J Biol Chem 275: 24500-24505, 2000.

19. Roy HK, Olusola BF, Clemens DL, Karolski WJ, Ratashak A, Lynch HT and Smyrk TC: AKT proto-oncogene overexpression is an early event during sporadic colon carcinogenesis. Carcinogenesis 23: 201-205, 2002

20. Saini MK and Sanyal SN: PTEN regulates apoptotic cell death through PI3-K/Akt/GSK3 $\beta$ signaling pathway in DMH induced early colon carcinogenesis in rat. Exp Mol Pathol 93: 135-146, 2012.

21. Xue G, Restuccia DF, Lan Q, Hynx D, Dirnhofer S, Hess D, Ruegg $\mathrm{C}$ and Hemmings BA: Akt/PKB-mediated phosphorylation of Twist 1 promotes tumor metastasis via mediating cross-talk between PI3K/Akt and TGF- $\beta$ signaling axes. Cancer Discov 2 : 248-259, 2012.

22. Li Y, Yang Q, Guan H, Shi B, Ji M and Hou P: ZNF677 suppresses Akt phosphorylation and tumorigenesis in thyroid cancer. Cancer Res 78: 5216-5228, 2018

23. Tang Y, Xiao G, Chen Y and Deng Y: LncRNA MALAT1 promotes migration and invasion of non-small-cell lung cancer by targeting miR-206 and activating Akt/mTOR signaling. Anticancer Drugs 29: 725-735, 2018.

24. Livak KJ and Schmittgen TD. Analysis of relative gene expression data using real-time quantitative PCR and the 2(-Delta Delta C(T)) method. Methods 25: 402-408, 2001.

25. Malkomes P, Lunger I, Luetticke A, Oppermann E, Haetscher N, Serve H, Holzer K, Bechstein WO and Rieger MA: Selective AKT Inhibition by MK-2206 represses colorectal cancer-initiating stem cells. Ann Surg Oncol 23: 2849-2857, 2016.

26. Wisinski KB, Tevaarwerk AJ, Burkard ME, Rampurwala M, Eickhoff J, Bell MC, Kolesar JM, Flynn C and Liu G: Phase I Study of an AKT Inhibitor (MK-2206) combined with lapatinib in adult solid tumors followed by dose expansion in advanced HER 2+ breast cancer. Clin Cancer Res 22: 2659-2667, 2016.

27. Agarwal E, Chaudhuri A, Leiphrakpam PD, Haferbier KL, Brattain MG and Chowdhury S: Akt inhibitor MK-2206 promotes anti-tumor activity and cell death by modulation of AIF and Ezrin in colorectal cancer. BMC Cancer 14: 145, 2014.
28. Baek KH: Cytokine-regulated protein degradation by the ubiquitination system. Curr Protein Pept Sci 7: 171-177, 2006.

29. Shin JM, Yoo KJ, Kim MS, Kim D and Baek KH: Hyaluronanand RNA-binding deubiquitinating enzymes of USP17 family members associated with cell viability. BMC Genomics 7: 292 , 2006.

30. Burrows JF, Kelvin AA, McFarlane C, Burden RE, McGrattan MJ, De la Vega M, Govender U, Quinn DJ, Dib K, Gadina M, et al: USP17 regulates Ras activation and cell proliferation by blocking RCE1 activity. J Biol Chem 284: 9587-9595, 2009.

31. Song C, Liu W and Li J: USP17 is upregulated in osteosarcoma and promotes cell proliferation, metastasis, and epithelial-mesenchymal transition through stabilizing SMAD4. Tumour Biol 39: $1010428317717138,2017$.

32. Wang M, He SF, Liu LL, Sun XX, Yang F, Ge Q, Wong WK and Meng JY: Potential role of ZEB1 as a DNA repair regulator in colorectal cancer cells revealed by cancer-associated promoter profiling. Oncol Rep 38: 1941-1948, 2017.

33. Kim J, D'Annibale S, Magliozzi R, Low TY, Jansen P, Shaltiel IA, Mohammed S, Heck AJ, Medema RH and Guardavaccaro D: USP17- and SCF $\beta$ TrCP-regulated degradation of DEC1 controls the DNA damage response. Mol Cell Biol 34: 4177-4185, 2014

34. Zhu J, Sun Y, Lu Y, Jiang X, Ma B, Yu L, Zhang J, Dong X and Zhang Q: Glaucocalyxin A exerts anticancer effect on osteosarcoma by inhibiting GLI1 nuclear translocation via regulating PI3K/Akt pathway. Cell Death Dis 9: 708, 2018.

35. Koundouros N and Poulogiannis G: Phosphoinositide 3-Kinase/Akt Signaling and Redox Metabolism in Cancer. Front Oncol 8: 160, 2018.

36. Zheng J, Zhang M, Zhang L, Ding X, Li W and Lu S: HSPC 159 promotes proliferation and metastasis by inducing epithelial-mesenchymal transition and activating the PI3K/Akt pathway in breast cancer. Cancer Sci 109: 2153-2163, 2018.

37. Zhong C, Chen Y, Tao B, Peng L, Peng T, Yang X, Xia X and Chen L: LIM and SH3 protein 1 regulates cell growth and chemosensitivity of human glioblastoma via the PI3K/AKT pathway. BMC Cancer 18: 722, 2018.

38. Gong T, Cui L, Wang H, Wang $\mathrm{H}$ and Han N: Knockdown of KLF5 suppresses hypoxia-induced resistance to cisplatin in NSCLC cells by regulating HIF-1 $\alpha$-dependent glycolysis through inactivation of the PI3K/Akt/mTOR pathway. J Transl Med 16: $164,2018$.

39. O'Reilly D, Johnson P and Buchanan PJ: Hypoxia induced cancer stem cell enrichment promotes resistance to androgen deprivation therapy in prostate cancer. Steroids 152: 108497, 2019.

40. Liu W, Yu WM, Zhang J, Chan RJ, Loh ML, Zhang Z, Bunting KD and Qu CK: Inhibition of the Gab2/PI3K/mTOR signaling ameliorates myeloid malignancy caused by Ptpn11 (Shp2) gain-of-function mutations. Leukemia 31: 1415-1422, 2017.

41. Wang L, Cao XX, Chen Q, Zhu TF, Zhu HG and Zheng L: DIXDC1 targets $\mathrm{p} 21$ and cyclin D1 via PI3K pathway activation to promote colon cancer cell proliferation. Cancer Sci 100: 1801-1808, 2009

42. Zhao W, Sun Q, Yu Z, Mao S, Jin Y, Li J, Jiang Z, Zhang Y, Chen M, Chen P, et al: MiR-320a-3p/ELF3 axis regulates cell metastasis and invasion in non-small cell lung cancer via PI3K/Akt pathway. Gene 670: 31-37, 2018

43. Zhao J, Xu J and Zhang R: SRPX2 regulates colon cancer cell metabolism by miR-192/215 via PI3K-Akt. Am J Transl Res 10: 483-490, 2018

44. Zhang B, Liu L, Guan H, Wang H, Zhang Z and Zhou P: HepG2 cell cycle related gene transcriptional profiles are altered by a novel vanillin derivative BVAN08. J Med Discov 2: 17036, 2017.

45. Deng N and Chen Y: Application of CRISPR-Cas9 gene editing system: Non-viral delivery strategies and improvements. J Med Discov 3: 17057, 2018.

This work is licensed under a Creative Commons Attribution-NonCommercial-NoDerivatives 4.0 International (CC BY-NC-ND 4.0) License. 